\title{
Complete obstruction of an endotracheal tube due to an unexpected blood clot in a patient with a hemo-pneumothorax after repositioning of the patient for lumbar spine surgery
}

\author{
Hyun Kyoung Lim ${ }^{1}$, Mi Hyeon Lee ${ }^{2}$, Hee Yong Shim ${ }^{1}$, Hyo Jin Byon ${ }^{1}$, and Hyun Soo Ahn ${ }^{1}$ \\ Department of Anesthesiology and Pain Medecine, ${ }^{1}$ Inha University Hospital, Incheon, ${ }^{2}$ Kangnam Sacred Heart Hospital, Hallym \\ University College of Medicine, Seoul, Korea
}

Endotracheal intubation is widely used to maintain the airway during general anesthesia. Intraoperative endotracheal tube obstruction can be caused by biting, kinking, external compression, and secretions or other intraluminal material [1]. We experienced a case of complete obstruction of the endotracheal tube due to a blood clot during surgery with a patient in the prone position who had a hemo-pneumothorax. A 40-year-old male with bilateral multiple rib fracture associated with a hemo-pneumothorax without hemoptysis, L2 spine fracture and left distal tibia medial malleolar fracture were the result of an accident in which the patient fell down 3 meters. On the seventh day of hospitalization, posterior lumbar interbody fusion was scheduled to correct the L2 spine fracture. Intubation was performed with an $8.0 \mathrm{~mm}$ armored tube, and vital signs were stable: blood pressure, 100-120 systolic and 60-70 diastolic; heart rate, 75-90 beats/min; the electrocardiogram showed a normal sinus rhythm with 99-100\% oxygen saturation: $\mathrm{ETCO}_{2}, 34-36 \mathrm{mmHg}$; peak inspiratory pressure, $18-22 \mathrm{cmH}_{2} \mathrm{O}$. After repositioning the patient for spinal surgery from the supine to the prone position, there was a slight increase in the peak inspiratory pressure to 23-25 $\mathrm{cmH}_{2} \mathrm{O}$, but there was no change in both lungs sound and in rib cage movement during mechanical ventilation. Thirty minutes after maintaining the patient in the prone position, a sudden rise in the peak inspiratory pressure and $\mathrm{ETCO}_{2}$ were detected at $32-35 \mathrm{cmH}_{2} \mathrm{O}$ and $43-45 \mathrm{mmHg}$, respectively, with a change in the capnograph indicating an obstructive pattern. Endotracheal suction was done immediately, but was unsuccessful in relieving the pressure. Mechanical ventilation was turned off and manual ventilation was performed, during which progressive increases in resistance were sensed. Eventually, the capnograph was flattened with a gradual decline in oxygen saturation to $75 \%$. Based on suspicion of endotracheal tube obstruction, a bronchoscopy was done using a flexible bronchoscope while intermittent manual ventilation with $100 \%$ $\mathrm{O}_{2}$ was carried out. In addition, we confirmed the presence of total occlusion of the endotracheal tube at the distal end by a blood clot. Without delay, the patient was turned back to the supine position and the endotracheal tube was extubated, at which point, the blood pressure was between 160-170 systolic and 95-110 diastolic; the heart rate was 120-130 beats/min and the oxygen saturation was at $45 \%$. Immediate mask ventilation was performed until pulse oximetry Oxygen saturation rose up to $95 \%$ and the patient was re-intubated with a $7.5 \mathrm{~mm}$ armored tube. The formally extubated tube was fully obstructed with a huge blood clot at the distal end to the cuff site (Fig. 1). The vital signs became stable after re-intubation, and the $\mathrm{ETCO}_{2}$ and peak inspiratory pressure were maintained between $34-36 \mathrm{mmHg}$ and $18-22 \mathrm{cmH}_{2} \mathrm{O}$, respectively. The surgery was completed without further incidence, and the patient was transferred to the general ward without any sequelae, and eventually discharged at POD 21.

There are several reported cases on airway obstruction due to blood clots. Arney et al. [2] reported on the cleansing,

Corresponding author: Hyun Kyoung Lim, M.D., Department of Anesthesiology and Pain Medecine, Inha University Hospital, 7-206, Sinheungdong 3-ga, Jung-gu, Incheon 400-711, Korea. Tel: 82-32-890-3968, Fax: 82-32-881-2476, E-mail: hkliman@inha.ac.kr

(c) This is an open-access article distributed under the terms of the Creative Commons Attribution Non-Commercial License (http:// creativecommons.org/licenses/by-nc/3.0/), which permits unrestricted non-commercial use, distribution, and reproduction in any medium, provided the original work is properly cited. 

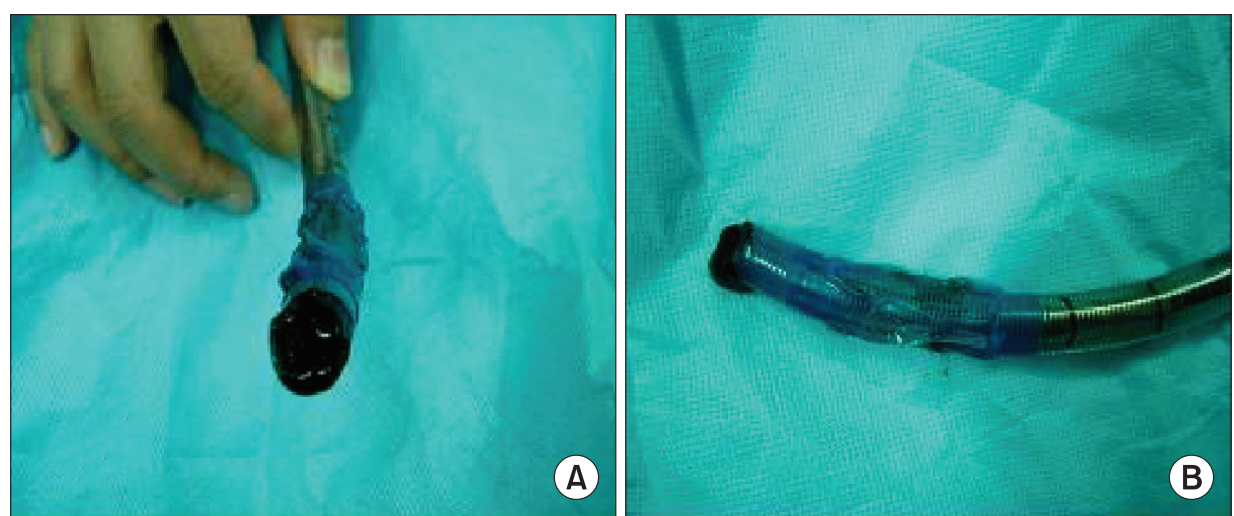

Fig. 1. (A) Anterior view of removed armored endotracheal tube showing complete obstruction of distal lumen due to impaction of blood clot. (B) Side view of removed armored endotracheal tube showing complete obstruction of distal lumen due to impaction of blood clot.

aspiration, removal, and careful use of thrombolytic agents after identifying a blood clot through direct bronchoscopy, when there was a partial airway obstruction due to endobronchial blood clot. Veronese et al. [3] reported a case, in which, a large blood clot in the endotracheal tube was removed by extubation without using invasive measures. Lin et al. [4] reported a case of acute endotracheal tube obstruction caused by unexpected hemoptysis in a patient with inactive tuberculosis undergoing spinal surgery in the prone position, which was relieved by just placing the patient back into the supine position allowing for direct visualization of the bleeding site with a flexible bronchoscopy. What we experienced was a case of endotracheal tube obstruction due to a blood clot in a patient with a traumatic hemo-pneumothorax without preoperative hemoptysis who underwent spinal surgery in the prone position.

In conclusion, when general anesthesia is performed in patients with a hemo-pneumothorax who need to be positioned in the prone position for the surgery, it is essential to consider the possibility of an endotracheal tube obstruction, and a tracheobronchial tree evaluation should be performed with a flexible bronchoscope for direct detection of bleeding sites and for the removal of any visible blood clots even in cases where there is no evidence of preoperative hemoptysis. Additionally, it should be kept in mind that a blood clot could migrate into the endotracheal tube due to gravitational force when the patient is moved into the prone position even though endotracheal suction was applied to eliminate secretions in the supine position.

\section{References}

1. Handerson J. Airway management in the adult. In: Miller's anesthesia. 7th ed. Edited by Miller RD: Philadelphia, Elsvier Churchill Livingstone. 2010, p 1601.

2. Arney KL, Judson MA, Sahn SA. Airway obstruction arising from blood clot: three reports and a review of the literature. Chest 1999; 115: 293-300.

3. Veronese S, Cutrone C, Innocente F, Ori C. Endotracheal tube and trachebronchial obstruction due to a large blood clot. Case report. Minerva Anesthesiol 2002; 68: 561-5.

4. Lin JA, Wong CS, Cherng $\mathrm{CH}$. Unexpected blood clot-induced acute airway obstruction in a patient with inactive pulmonary tuberculosis during lumbar spine surgery in the prone position--a case report. Acta Anaesthesiol Taiwan 2005; 43: 93-7. 